\title{
Mixed Zone between Drug-Induced Hyperpigmentation and Generalized Fixed Pigmented Erythema
}

\section{Tarik Hanafi ${ }^{1 *}$, Amine Essaoudi ${ }^{2}$, Hicham Titou ${ }^{1}$, Hasna Kerrouch ${ }^{1}$, Rachid Frikh ${ }^{1}$, Naoufal Hjira ${ }^{1}$ and Mohammed Boui ${ }^{1}$}

\author{
${ }^{1}$ Department of Dermatology and Venereology, Mohammed V Military Hospital, Faculty of \\ Medicine and Pharmacy of Rabat, Mohammed V University, Morocco \\ ${ }^{2}$ Pathology Department, Mohammed V Military Hospital, Faculty of Medicine and Pharmacy of \\ Rabat, Mohammed V University, Morocco
}

*Corresponding author: Tarik Hanafi, Department of Dermatology and Venereology, Mohammed V Military Hospital, Faculty of Medicine and Pharmacy of Rabat, Mohammed V University, Hay Riad 10000 Rabat, Morocco

\begin{abstract}
We report a particular case of fixed drug eruption (FDE), in its erythematous and pigmented form, as a fixed pigmented erythema (FPE), which manifests in a generalized form, evolving for three years, and discuss the imputability to piroxicam and the Ramipril/Hydrochlorothiazide combination. Indeed, chronological, semiological, bibliographic criteria, were imprecise and non-discriminatory. We ended up retaining the diagnosis of fixed pigmented erythema (FPE) of particular diffuse presentation induced probably by piroxicam, based on clinical, histological and evolutive criteria. However, it remains a mixed zone between drug-induced hyperpigmentation and generalized fixed pigmented erythema with diffuse hypermelanosis.
\end{abstract}

\section{Keywords}

Fixed pigmented erythema, Drug-induced hyperpigmentation, Piroxicam and Hydrochlorothiazide

\section{Introduction}

The first case of fixed pigmented erythema [FPE] described in the literature dates back to 1894 by Brocq, reporting a case of pigmented evolutionary erythema induced by antipyrine [1]. It is a benign drug reaction induced by different therapeutic classes mainly, NSAIDs and antibiotics [2].

We report a particular case of $\mathrm{FPE}$, in a generalized and extensive form evolving for three years, and discuss the diagnostic balance between, hyper- pigmentation as toxiderma and generalized fixed pigmented erythema presenting with diffuse hypermelanosis, we will also discuss, the imputability compared to piroxicam and the Ramipril/Hydrochlorothiazide combination.

\section{Case Report}

He is a 56-year-old patient, followed for three years for high blood pressure under the combination of Ramipril/Hydrochlorothiazide $10 \mathrm{mg} / 25 \mathrm{mg}$ and arthritis of the knee treated with Piroxicam $20 \mathrm{mg} / \mathrm{d}$ with prolonged self-medication without medical supervision. The patient presented with an eruption evolving for approximately 3 years, characterized by confluent macules in erythematous and pruriginous layers with progressive extension and evolving towards a residual brownish diffuse discreetly pruriginous hyperpigmentation, affecting the four limbs, thighs and arms, the suprapubic region, the inguinal and axillary region, as well as the neck, this hyperpigmentation is accentuated in arms, lateral face of the trunk and gluteal region. Lesions were in an inflammatory phase in the axillary folds and absent in face, forearms, hands, back and the oral and genital mucosa (Figure 1a and Figure 1b).

The biological assessment in particular, blood count, blood ionogram, ASAT-ALAT, urea-creatinine,

Citation: Hanafi T, Essaoudi A, Titou H, Kerrouch H, Frikh R, et al. (2020) Mixed Zone between DrugInduced Hyperpigmentation and Generalized Fixed Pigmented Erythema. Clin Med Rev Case Rep 7:296. doi.org/10.23937/2378-3656/1410296

Accepted: January 29, 2020: Published: January 31, 2020

Copyright: (C) 2020 Hanafi T, et al. This is an open-access article distributed under the terms of the Creative Commons Attribution License, which permits unrestricted use, distribution, and reproduction in any medium, provided the original author and source are credited. 


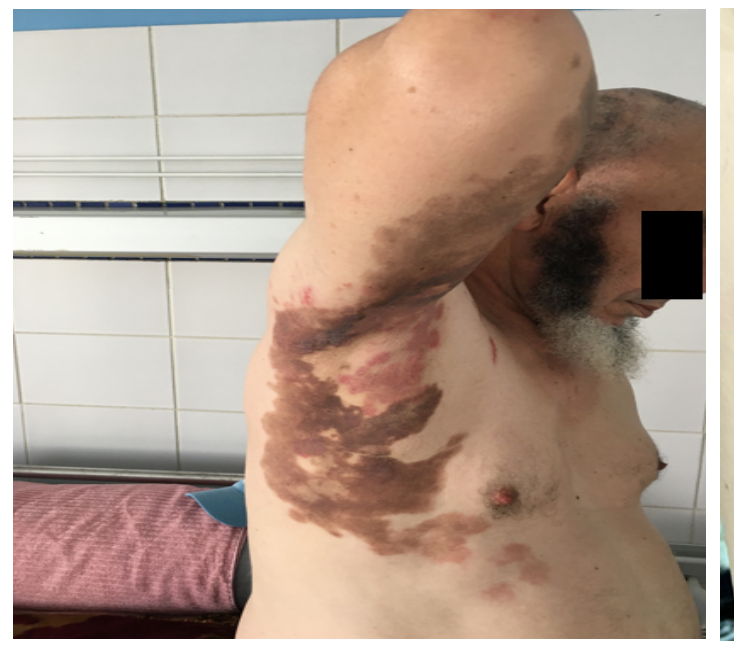

A

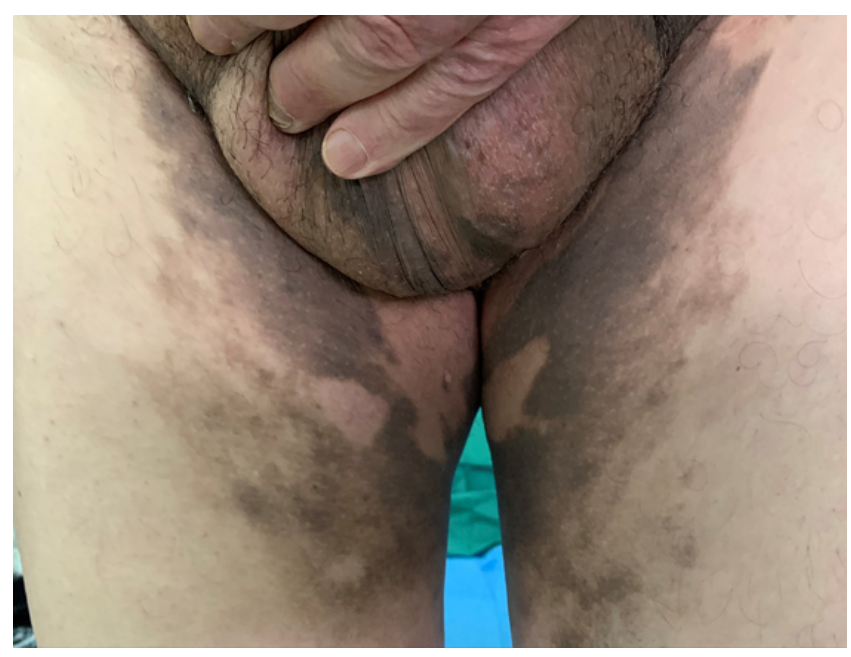

B

Figure 1: a,b) Confluent macules in erythematous and pruriginous layers with progressive extension and evolving towards a residual brownish diffuse discreetly pruriginous hyperpigmentation accentuated in arms, trunk and gluteal region, Lesions are in an inflammatory phase in the axillary folds.
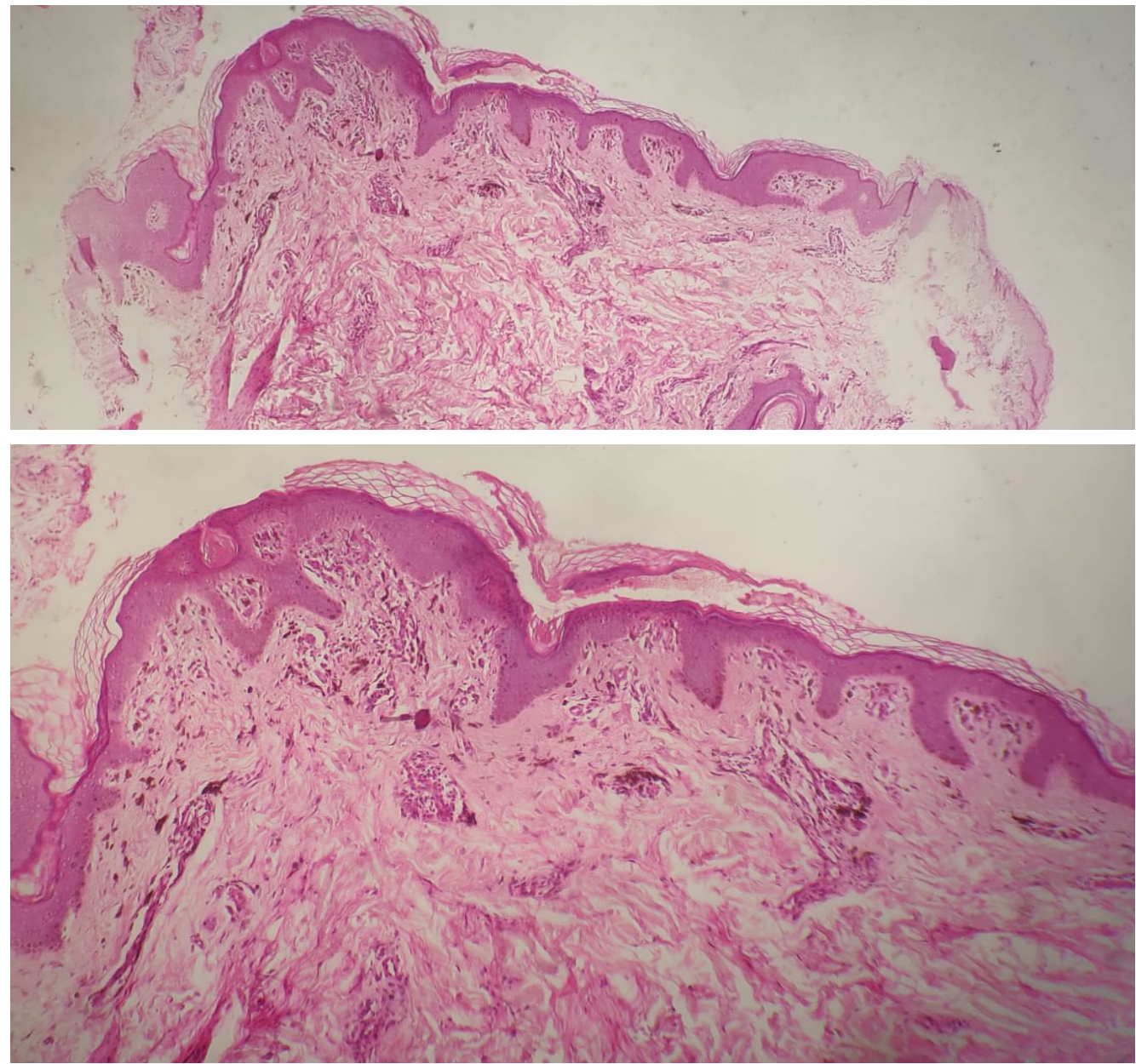

Figure 2: Interface obscuring dermoepidermal junction with mélanophages, eosinophils and mixed infiltrate (a) Hematoxylin-Eosin, × 10; b) Hematoxylin-Eosin, × 25).

cortisolemia, TSHUS and anti TPO ac were without abnormalities apart from hypokalemia at $3.1 \mathrm{mmol} / \mathrm{I}$. The diagnosis of pigmentogenic lichen, Drug-induced skin pigmentation or ashy dermatosis was suspected first, however, symmetrical drug-related inter- triginous and flexural exanthema (SDRIFE) remains a plausible diagnosis.

A skin biopsy was performed showing an interface obscuring dermoepidermal junction with mélanophages, eosinophils and mixed infiltrate (Hematoxy- 
lin-Eosin, Figure 2a $(\times 10)$ and Figure 2b $(\times 25)$. After piroxicam was stopped, itching and the extension of hyperpigmentation were stopped, and no new inflammatory lesions were observed. Despite stopping Piroxicam and a three-month topical depigmenting treatment, we have not noticed any improvement in hyperpigmentation.

\section{Discussion}

FPE is clinically characterized by the presence of single or multiple lesions, round or oval, with a well-defined border and residual pigmented development, which allow even in the presence of scar lesions to make the diagnosis [3].

The pathogenesis probably involves the interaction of part of the offending drug acting as a hapten triggering an immune response involving memory $T$ cells residing in the skin. This toxiderma is due to delayed cellular hypersensitivity in which the immune memory mediated by CD8+ T cell remains localized. The lesions always recur at the same sites where immune memory is located [4]. However, continuous and repeated exposure may aggravate the rash at specific anatomical sites and may lead to the progressive involvement of new sites with each exposure [2].

The first case reporting a generalized form of FPE was described in 1984 in a young patient of North African origin and was linked to a polypharmacy including NSAIDs [5]. In 2018, Kornmehl, et al. proposed a small review of the literature [6], antibiotics (50\%) and nonsteroidal anti-inflammatory drugs (36\%) were the most accused, the number of sites affected may increase with re-exposure, Thus the FPE can be presented in diffuse form. On the other hand, some authors describe a particular presentation of the FPE performing diffuse generalized hypermelanosis [7].

We preferred to qualify the reported case as being generalized, in opposition to the pathogenic assimilation typically correlated with localized immune stimulation and classically described in common FPE, but also because of the multi-site localisations, and the frankly extensive character of the lesions.

However, SDRIFE [8] remains an important differential diagnosis, we didn't retain it because of, the presence of pruritus, the fact that the point of departure of the lesions was not at the folds, as shown in the Figure 1 , recent lesions have settled in the axillary folds surrounded by old pigmented lesions, and the topography which is not limited to the flexion zones.

Otherwise, on the pharmaceutical side, Piroxicam has been implicated in several observations [9-11] unlike Ramipril and Hydrochlorothiazide, indeed antihypertensive treatments are more often linked to photodistributed hyperpigmentations [12] which is not the case in our patient.
The importance of itching and the absence of the photodistributed character, resolution after stopping piroxicam and the histological image seemed to us to favor the diagnosis of FPE with particular diffuse presentation.

However, for concrete discrimination, it is recommended to carry out drug patch tests on the site previously affected by FPE, this seems even more interesting, when several molecules are attributable $[4,13,14]$.

\section{Conclusion}

Chronological, semiological, bibliographic criteria, were imprecise and non-discriminatory. We support the diagnosis of fixed pigmented erythema of particular diffuse presentation induced by piroxicam, based on clinical, histological and evolutive criteria. However, it remains a mixed zone between hyperpigmentation as toxiderma and generalized fixed pigmented erythema presenting with diffuse hypermelanosis.

\section{Conflicts of Interest}

None.

\section{References}

1. Brocq $L$ (1894) Eruption erythémato-pigmentée fixé due à l'antipyrine. Ann Dermatol Syphiligr (Paris) 5: 308-313.

2. Chang AY (2018) Fixed Drug Eruption. In: Rosenbach M, Wanat K, Micheletti R, Taylor L, Inpatient Dermatology. Springer, Cham.

3. Valeyrie-Allanore L, Lebrun-Vignes B, Bensaid B, Sassolas $B$, Barbaud $A$ (2015) Érythème pigmenté fixe : épidémiologie, physiopathologie, clinique, diagnostic différentiel et modalités de prise en charge. Ann Dermatol Venereol 142: 701-706.

4. A Barbaud, Groupe FISARD de la SFD (2018) Allergological investigations in fixed pigmented erythema. Method recommended by the FISARD (drug eruptions) group of the French Dermatology Society. Annales de Dermatologie et de Vénéréologie 145: 210-213.

5. Prigent F (1984) Fixed pigmented erythema: Generalized form. Rev Med Interne 5: 159-160.

6. Kornmehl H, Gorouhi F, Konia T, Fung MA, Tartar DM (2018) Generalized fixed drug eruption to piperacillin/tazobactam and review of literature. Dermatol Online J 24.

7. Mahboob A, Haroon TS (1998) Drugs causing fixed eruptions: A study of 450 cases. Int J Dermatol 37: 833-838.

8. Winnicki M, Shear NH (2011) A systematic approach to systemic contact dermatitis and symmetric drug-related intertriginous and flexural exanthema (SDRIFE): A closer look at these conditions and an approach to intertriginous eruptions. Am J Clin Dermatol 12: 171-180.

9. Cuerda Galindo E, Goday Buján JJ, García Silva JM, Martínez W, Verea Hernando M, et al. (2004) Fixed drug eruption from piroxicam. J Eur Acad Dermatol Venereol 18: 586-587.

10. Hajar Imtihani, Vika Fintaru, Jeffrey Giantoro, Niken Indrastuti, Fajar Waskito (2019) Multiple Fixed Drug Eruption Due to Piroxicam: A Brief Case Report. Dermatology Case Reports Rep 3: 2. 
11. B Fernández-Jorge, JJ Goday, M Almagro, E Fonseca (2008) Fixed Drug Eruption Due to Piroxicam. Actas Dermosifiliogr 99: 239-240.

12. Rosa Giménez-Garcia (2016) Hyperpigmentation induced by combination therapy with Telmisartan Hydrochlorothiazide. The Journal of Clinical Hypertension 18: 361-362.
13. Alanko K (1994) Topical provocation of fixed drug eruption. A study of 30 patients. Contact Dermatitis 31: 25-27.

14. Andrade P, Brinca A, Goncalo M (2011) Patch testing in fixed drug eruptions-a 20-year review. Contact Dermatitis 65: 195-201. 